\title{
When Severe Preeclampsia Met Pulmonary Arterial Hypertension: If Time Goes Back
}

\author{
Zhiliang Chen ${ }^{1}$, Yuqi Liu ${ }^{2}$, Guoliang Tan ${ }^{1}$, Deyuan You ${ }^{3}$, and Xuri Sun ${ }^{1}$ \\ ${ }^{1}$ Second Affiliated Hospital of Fujian Medical University \\ ${ }^{2}$ Second affiliated hospital of Fujian Medical University \\ ${ }^{3}$ Affiliation not available
}

May 5, 2020

\begin{abstract}
Once again, we look back at a case of a maternal who died five years ago with severe preeclampsia combined with PAH. We thought if we were familiar with the special pathophysiological mechanism of PAH and adopt proper treatment methods at that time, her death might be avoided.
\end{abstract}

\section{Case}

A 23-year-old G2P0 woman with a body mass index (BMI) of $29 \mathrm{~kg} / \mathrm{m} 2$ was admitted at $333 / 7$ weeks of gestation. She reported a 7-day history of lower limb edema and 3-day history of dizziness. Her initial physical condition was as follows: alert, body temperature $\left(36.5^{\circ} \mathrm{C}\right)$, respiratory rate $(22 \mathrm{~b} . \mathrm{p} . \mathrm{m})$, heart rate (88 b.p.m), blood pressure $(135 / 97 \mathrm{mmHg}), \mathrm{SaO}_{2}$ was $93 \%$. Bilateral fingers were clubbing fingers, with edema of both lower limbs $(+++)$. Peripheral blood tests disclosed with hemoglobin and hematocrit levels of $16.5 \mathrm{~g} / \mathrm{dL}$ and $57 \%$, respectively, a platelet count of $77 \times 10^{3} / \mu \mathrm{L}$ and white blood cell count $12.2 \times 10^{3} / \mu \mathrm{L}$. Additionally, laboratory data of serum revealed albumin $22.8 \mathrm{~g} / \mathrm{L}$, lactate dehydrogenase $488 \mathrm{IU} / \mathrm{L}$, urea nitrogen, creatinine, blood glucose and electrolytes were normal. Her arterial blood gas analysis showed $\mathrm{pH}$ 7.38, $\mathrm{PaO}_{2} 93 \mathrm{mmHg}\left(\mathrm{FiO}_{2} 0.4\right), \mathrm{PaCO}_{2} 32 \mathrm{mmHg}$, standard bicarbonate $22.5 \mathrm{mmol} / \mathrm{L}$ and base deficit of -1.7 $\mathrm{mEq} / \mathrm{L}$. Her urine protein $(++)$. Ultrasound showed that the fetal growth retardation, the age of head and femur both were 29 weeks.

The patient was admitted to the obstetric ward and was initially diagnosed as severe preeclampsia, partial hemolytic anemia, elevated liver function and low platelet count (HELLP) syndrome, hypoproteinemia, cardiac insufficiency and intrauterine growth restriction. After 18 hours of admission, due to the deteriorating situation of breath and saturation, she was performed "cesarean section in the lower uterine segment". During the operation, $1500 \mathrm{ml}$ was put in and $900 \mathrm{ml}$ was taken out, bleeding only $150 \mathrm{ml}$, the blood oxygen saturation fluctuated from $80 \%$ to $90 \%$.

The patient was admitted into intensive care unit (ICU)after operation with heart rate 98 b.p.m, blood pressure 117/90 $\mathrm{mmHg}$, oxygen saturation $85 \%$ and central venous pressure $(\mathrm{CVP}) 12 \mathrm{cmH}_{2} \mathrm{O}$. She accepted invasive ventilation and strengthening heart, diuresis and vasodilation management according the diagnosis of cardiac insufficiency, the initial ventilator parameter setting was respiratory rate 20 b.p.m,pressure support was $16 \mathrm{cmH}_{2} \mathrm{O}$, positive end expiratory pressure (PEEP) $10 \mathrm{cmH}_{2} 0$, fraction of inspiratory oxygen $\left(\mathrm{FiO}_{2}\right)$ was $90 \%$. On the day of operation, her fluid balance was $-500 \mathrm{ml}$ in ICU (13 hours).

In the first day after operation, her heart rate 102 b.p.m, blood pressure 100/70 $\mathrm{mmHg}$, oxygen saturation maintained between $75 \%$ and $85 \%$ with the same ventilator parameters and CVP $12 \mathrm{cmH}_{2} \mathrm{O}$. A color Doppler 
echocardiography was done to her, which indicated that the right atrium was $47 \mathrm{~mm} * 45 \mathrm{~mm}$, the diameter of the right ventricle and the aorta was separately $38 \mathrm{~mm}$ and $26 \mathrm{~mm}$, the diameter of the left ventricle was smaller, the ventricular septum and the free wall of the left ventricle moved in the same direction. No effusion was found in pericardial cavity. Doppler ultrasound showed that there was a little regurgitation of pulmonary valve and tricuspid valve, and the systolic pressure of pulmonary artery was $70 \mathrm{mmHg}$. Combined with the high hemoglobin(16.5 g/dL in admission)and clubbing fingers, we supplemented the diagnosis of chronic severe pulmonary hypertension and down regulated PEEP to $6 \mathrm{cmH} 20$ as well as strengthen dehydration and the liquid limitation. In the first day after operation, her fluid balance was $-2800 \mathrm{ml}$.

In the morning of second day after operation, the patient's blood pressure began to down slowly, her arterial blood gas analysis showed $\mathrm{pH}$ 7.44, $\mathrm{PaO}_{2} 48 \mathrm{mmHg}\left(\mathrm{FiO}_{2}\right.$ 1.0), $\mathrm{PaCO}_{2} 36 \mathrm{mmHg}$, standard bicarbonate $25.8 \mathrm{mmol} / \mathrm{L}$ and base deficit of $1.2 \mathrm{mEq} / \mathrm{L}$, lactic acid $1.5 \mathrm{mmol} / \mathrm{L}$; her peripheral blood tests showed white blood cell count rose to $15.2 \times 10^{3} / \mu \mathrm{L}$ with NE74.4\%, hemoglobin and platelet count down to $108 \mathrm{~g} / \mathrm{dL}$ with hematocrit $36 \%$ and $65 \times 10^{3} / \mu \mathrm{L}$. Considered that the patient's blood pressure was only about $90 / 70 \mathrm{mmHg}$ and CVP still $12 \mathrm{cmH}_{2} \mathrm{O}$, we reduced the dosage of diuretics to slow down the rate of negative liquid balance. 6 hours later, she died of a sudden cardiac arrest. In the second day after operation, her fluid balance was $-600 \mathrm{ml}$ (8 hours).

\section{Discussion}

Pulmonary hypertension is one of the most serious complications of parturient women. Because of special pathophysiological mechanism, there are some different treatments on liquid balance, anesthesia, operation, postoperative treatment and ventilator settings. A study on the outcome of 151 women with pulmonary hypertension showed that although the general mortality rate dropped to $4.6 \%$ ( 7 / 151), the mortality rate of idiopathic pulmonary arterial hypertension (iPAH) was still as high as $42.9 \%(3 / 7)^{[1]}$. PAH is the first group of pulmonary hypertension classification, with higher risk of complications and mortality ${ }^{[2]}$. Because of the atypical clinical manifestations of $\mathrm{PAH}$, clinicians should have enough vigilance to find clues and make early diagnosis, which is the most important first step for the survival of women with PAH ${ }^{[3]}$.

This was a case of maternal death of PAH 5 years ago. She was diagnosed HELLP syndrome and her possible cause of death was considered pulmonary embolism. We thought her death was inevitable at that time ${ }^{[4]}$. Now looking back again, we found there were some obvious defects and errors in the diagnosis and treatment on the maternal and it was necessary to discuss it again. If time goes back, maybe she would avoid death.

First of all, although the patient did not diagnose PAH during pregnancy, she had obvious dizziness and clubbing fingers, hypoxemia and increased hemoglobin. An experienced doctor should suspect the underlying disease of cardiovascular or lung basic diseases and immediately give a color Doppler echocardiography to find out the cause. The patient did color Doppler echocardiography on the first day after the operation and showed an obvious manifestations of high load of right ventricle, such as right atrium and right ventricle enlargement, tricuspid valve and pulmonary valve regurgitation, left ventricle compression becoming smaller, ventricular septum and left ventricular free wall moving in the same direction, pulmonary artery pressure rose to $70 \mathrm{mmHg}$. It was no doubt that the diagnosis of severe pulmonary hypertension. We considered she was an iPAH.

PAH patients have a special pathophysiological mechanism, progressive right heart failure is the most common cause of deterioration ${ }^{[3]}$. In patients with severe right heart insufficiency, the preload reserve function of the right heart is significantly reduced, and the tension-free or low tension stage is significantly shortened or disappeared, a very small amount of fluid change may lead to a significant change in cardiac output and a large fluctuation in hemodynamics ${ }^{[5]}$. Because of the underlying disease of $\mathrm{PAH}$, positive fluid balance and positive pressure ventilation during operation cause acute aggravation of right precordial and afterload. The increase of right ventricular pressure compressed the left ventricle through the interventricular septum, the decrease of left ventricular volume led to the limitation of diastolic function, the decrease of cardiac output, and then the decrease of blood pressure and oxygenation. The spasm of pulmonary artery because of the low blood pressure and oxygenation aggravated the pulmonary hypertension to enter the vicious circle of 
autonomic deterioration.

If time goes back, based on our current understanding of PAH and the application of critical ultrasound technology, patients will be given cardiac ultrasound examination immediately after admission to make an early diagnosis of PAH; obstetricians may be more active in choosing to terminate pregnancy as soon as possible; anesthesiologists may not choose general anesthesia and will be more strict in controlling intraoperative fluid intake, because a study have suggested an associated between general anesthesia and maternal mortality in women receiving general compared with neuraxial anesthesia for delivery ${ }^{[6]}$; ICU physicians may strengthen diuretic treatment or even choose continuous blood purification treatment, as well as a lower PEEP, which was a "domino effect" caused by untimely diagnosis because of the doctors were not alert to PAH.

Secondly, the first week after operation is the peak period of death of PAH maternal, obviously the ICU physicians at that time were inexperienced in this respect. The patient's blood pressure dropped on the second day after operation, which was exactly the manifestation of low cardiac output caused by right ventricular overload and compression of left ventricle. Low blood pressure was the most serious situation of right heart failure. If time goes back, we will closely monitor echocardiography to dynamically guide the liquid treatment, increase the dosage of diuretics or actively apply continuous blood purification to reduce the right heart load and to promote the extent of reverse fluid resuscitation. Prone position ventilation can reduce the pulmonary artery pressure and extracorporeal membrane oxygenation(ECMO) treatment can improve oxygenation and pulmonary artery spasm, which would also be considered.

Unfortunately, this patient had severe preeclampsia with hypoproteinemia and partial HELLP syndrome. It has been reported that the proportion of $\mathrm{PAH}$ with severe preeclampsia was as high as $6.7 \%$. ${ }^{[7]}$. On the first day after the operation, the patient's hemoglobin decreased, but there was no obstetric hemorrhage and serious infection. At that time, we explained the decrease of hemoglobin by Hellp syndrome. If time goes back,we will also consider maybe hemodilution was another reason for a dropped hemoglobin level, because of the change of postpartum hormone level and the release of abdominal pressure as well as the fluid of peripheral tissue edema in severe preeclampsia returned to the great vessels. This kind of internal liquid infusion quietly increased the preload of right heart and misled clinicians to set a small goal for negative fluid balance in patients. In the second day after operation, due to the insufficient consideration of endogenous fluid reflux in patients with severe preeclampsia, the wrong decision to reduce the dosage of diuretics accelerated the patients' right heart failure and death.

Although the WHO against pregnancy for PAH women, the actual situation was that PAH women had a significant increase in the trend ${ }^{[3]}$. Although time can not goes back, the experiences of this case five years ago will help us more better in the next time. In summary, when severe preeclampsia met PAH, clinicians should strengthen their vigilance in the early diagnosis of $\mathrm{PAH}$, be familiar with the pathophysiological characteristics of right heart failure, do a good job on reverse fluid resuscitation, especially should enlarge the amount of negative fluid balance considered the endogenous fluid transfer; strengthen the close monitoring and treatment of hemodynamics guided by intensive ultrasound; emphasize a multidisciplinary team(MDT) that including obstetricians, anesthesiologist, ICU physician and cardiologist.

Patient's consent: We have obtained the permission to publication from the patient's family.

\section{REFERENCES}

[1] Karen Sliwa, Iris M. van Hagen, Werner Budts, Lorna Swan, Gianfranco Sinagra et al. Pulmonary hypertension and pregnancy outcomes: data from the registry of pregnancy and cardiac disease (ROPAC) of the European society of cardiology. European Journal of Heart Failure. 2016, 18, 1119-1128

[2] Meng ML, Landau R, Viktorsdottir O, Banayan J, Grant T, et al. Pulmonary hypertension in pregnancy: a report of 49 cases at four tertiary North American sites. Obstet Gynecol 2017,129:511-20.

[3] Stephanie R. Martin, Alexandra Edwards. Pulmonary hypertension and pregnancy. Obstetrics \& Gynecology. 2019, 134(5): 974-987. 
[4] Liu Yuqi, Guoliang Tan, Shang Chengming, Sun Xuri. The ICU is becoming a mian battlefield for severe maternal rescure in China: an 8-year single center clinical experience. CCM. 2017, 45(11): e1106-1110

[5] Pinsky MR. My paper 20 years later: effect of positive end-expiratory pressure on right ventricular function in humans. .Intensive Care Med,. 2014, 40(7): 935-941

[6] Jais X, Olsson KM, Barbera JA, Blanco I, Torbicki A, et al. Pregnancy outcomes in pulmonary arterial hypertension in the modern management era. Eur Respire J 2012, 40:881-5.

[7] Erin Thomas, Jie Yang, Jianjin Xu, Fabio V. Lima, Kathleen Stergiopoulos. Pulmonary hypertension and pregnancy outcomes: insights from the national inpatient sample. J Am Heart Assoc. 2017, 6: e006144-6156 